\title{
Inclusive learning strategies to enhance reading skill among the students with reading disability: An Occupation and Participation Approach to Reading Intervention (OPARI) in the rural Indian classroom
}

\author{
D. Annuncy Vinoliya ${ }^{1} \&$ Dr. R. Joseph Ponniah ${ }^{2}$ \\ ${ }^{1}$ Research Scholar, Department of Humanities and Social Sciences, National Institute of \\ Technology, Tiruchirappalli, Orchid Id: 0000-0003-0845-9687. \\ Email Id: annuncydavid.nitt@gmail.com, \\ 2Professor, Department of Humanities and Social Sciences, National Institute of Technology, \\ Tiruchirappalli. Orchid Id: 0000-0002-0618-6788. Email Id: Joseph@nitt.edu
}

\begin{abstract}
Reading is a challenging task for reading disability for which they need comprehensive strategies like sensory and neurocognitive requirements. With this notion, the article aims to find, the appropriate pedagogies and clinical practices used for intervening the reading disability in Indian public schools. To examine, qualitative interviews were conducted with ten high school teachers and four special education teachers, who work in the Government schools in India especially in the state of Tamil Nadu. The interview focused the opinions of the teachers on reading disability, facilities and pedagogies provided to the reading disability and from the interview, the study has derived the results in three main themes as, teachers' views on reading disabilities, inadequate teaching strategies for reading disabilities, special education to the reading disabilities. In the discussion section, the article attempts to resolve the issues raised in the interview by introducing an exclusive approach to intervene reading disability. The article incorporates the principles of the Occupational Participation and Adaptation to Reading Intervention approach (OPARI) to intervene the reading disability and attempts to find a solution to the issues. In addition, the article attempts to justify the neuroscience behind the OPARI by highlighting the dopamine activation in the brain while adapting reading. In the conclusion section, the article emphasizes therapeutic associative teaching and the need to implement OPARI in Indian classrooms.
\end{abstract}

Keywords: Reading disability, OPARI, Rural Indian classroom

\section{Introduction}

The hallmark of academic brilliance is often characterized by an individual's level of cognitive complexity to comprehend and construe through reading. Reading is an amalgamation of both cognitive and sensory rudiments that comprises of identification of letters, sound units, words,

This Open Access article is published under a Creative Commons Attribution Non-Commercial 4.0 International License (http://creativecommons.org/licenses/by-nc/4.0/), which permits non-commercial re-use, distribution, and reproduction in any medium, provided the original work is properly cited. For citation use the DOI. For commercial re-use, please contact editor@rupkatha.com. 
meaning, and comprehension (Sanocki \& Dyson, 2012; Zari \& Hasselhorn, 2021). Children get acquainted with reading during elementary education and failing to achieve the above-mentioned requisites for reading have projected children as reading disability; The reading disability demand comprehensive remedial measures to overcome it. Academicians and scientists have sought to find suitable clinical and pedagogical interventions for reading disabilities and have proposed that the comprehensive intervention i.e., pedagogical and therapeutic remediation could ameliorate reading. Educational Pedagogy interwoven with therapeutic intervention would stabilize the internal deformities in the brain regions that cause reading disability. To fulfil the demand of comprehensive intervention, the article employs occupational therapy. Occupational therapy is a form of healing, that intends to cure the physical and psychiatric condition of the individual or group through a desirable environment and daily activities. The term occupation refers to the activities used for the therapy (Occupational Therapy Practice Framework: Domain \& Process, 2014). It is a multidimensional model that works on the intrinsic and extrinsic factors of a participant and such therapeutic intervention is employed in professions like education, medicine, and other social communities. For instance, occupational therapists are available in every school in the United States of America who work along with teachers towards integrated education (Bissell \& Cermak, 2015). This therapy employs an explicit reading approach called Occupation and Participation Approach to Reading Intervention (OPARI), which nurtures reading through the desired medium.

After a close analysis of the intervention provided for reading disabilities in rural Indian schools, this article suggests launching a hard-core pedagogical and therapeutic intervention for reading disability.

\section{Background of the study}

In India, the percentage of students with reading disability among school-going children is roughly around $11.2 \%$ (Shetty \& Rai, 2014) along with the estimated prevalence of dyslexia to be around 5\%-12\% (Singh Charan \& Kaur, 2017). Reading disability is included in the Integrated Education for Disabled at the Secondary Stage (IEDSS). Under this scheme, proper education and intervention should be given for reading disability. But the provisions have only been provided for other special education needs and the provisions for students with reading disability are being cast aside. Therefore, the intervention for reading disabilities becomes questionable.

- "Is there any intervention given to the students with reading disability at the early stage?"

- "Do reading disabilities overcome the barriers in reading in inclusive education?"

- "Do teachers and special education trainers follow any strategies to teach students with reading disability?"

To answer these unanswered questions, a reading approach like OPARI takes an initiative to study the opinion, attitudes, and practices for students with a reading disability in rural Indian schools.

\section{Participants}

The study recruited participants using the top-down model comprising from the head person of the school to the high school teachers working in Rural Public high schools in Tamil Nadu, India. 
Along with them, special education teachers who work at the Block Resource Center in each district of Tamil Nadu in India are included in the study. The study includes ten high school teachers and four special education teachers. The participants were unknown to the interviewer before the initial contact and were randomly selected from the list of schools provided online by the district education office. The participants were met formally in their respective schools on producing the permission letter by the interviewer for this study. Similarly, the interviewer visited the Block Resource Centres that function at the district education office and met the special education teachers. The special education teachers have also been informed about the purpose of the study and the permission letter by the interviewer was produced to them.

\section{Methods}

Opting for a descriptive design enables us to arrive at a deeper understanding of the study and formulate a possible solution to the problem statement. The qualitative method has been employed in this study where the semi-structured interview has been used as a research strategy to elicit a piece of holistic information on the opinions and attitudes towards reading disabilities.

\section{Data Collection}

The interviews were conducted with ten mainstream teachers and four special education teachers. The one-to-one interview was conducted for about 20-30 minutes. The interviews were recorded and transcribed verbatim. All identifying information like the name of the participant, school, and geographical location was removed. Transcripts were analysed by both the first author and the second author. The transcripts were identified, grouped and highlighted according to the questions asked during the interview. The statements collected in the interview were consolidated into three themes: i.) teachers' views on reading disability, ii.) inadequate teaching strategies for students with reading disability and iii.) special education to the students with reading disability.

\section{Findings}

\section{Teachers' views on reading disabilities}

All the participants have at least three students with a reading disability in their classroom and the participants shared their views on the said disability. Participants 1, 7 and 10 felt that the class strength was huge and concentrating on students with reading disability is quite challenging. With regards to the opinion on students with a reading disability, participants 2, 6 and 9 stated that, as there was no early intervention, students struggle to pick up reading and writing in high schools and participant 2 has shared that the students with reading disability are good at oral skills but lack the ability to read and write with ease.

"They are good listeners, good at verbal and interpersonal communication but they are poor in doing homework and other written tasks, this is due to their disability in reading; but we teachers initially thought it as laziness towards reading and writing".

Almost all the participants had admitted that their primary concern in the inclusive classroom was students with other disabilities like visual impairment, hearing impairment and autism whereas reading disability i.e, dyslexia was a secondary concern to them. Participant 3 revealed the current scenario in the educational system in which the government legislation states, "All the students should be promoted till grade nine and none of them should not be demoted". 
As this rule exists, students with reading disabilities are promoted till grade nine and they meet the consequences in the high school final examinations. Eventually, some students with reading disabilities fail the exams and become clueless about their higher studies.

\section{Inadequate Teaching Strategies for reading disabilities}

Most of the participants said that they use the "rote strategy" to help such students to get through their exams and the teachers focused more on the topics which will have more weightage of marks in the examinations and as a result, they lack the required comprehension skills. Participants 5 and 7 stated that an hour per week has been allotted as Creative Learning Hour (CLH) in the curriculum for the students and the teachers would make use of that hour for teaching students with reading disability for the exams, leaving them with no exposure to tasks on creativity in the CLH. Four participants shared that they chose certain units in the syllabus for the special education needs and made the students to learn those chosen units. As a consequence, students with reading disabilities do not learn the entire topics in the curriculum. Participants 4 and 8 inform that they hardly use ICT like overhead projectors for teaching in the inclusive classroom and so ICT learning is not sufficient for the reading disability. The participants said that the classroom is modified in such a way to help the students with reading disability. Seating arrangements were such that the students occupied the first row so that they could listen to the class effectively. Participants 6 and 9 mentioned that they arranged the seating for learners with special education needs in which students with reading disability were seated along with toppers of the class as the teachers believed that peer teaching would help in better learning. What the teachers failed to understand was that the problem is with reading and writing and not with listening. Almost all the participants confessed that they are unaware of the educational neuroscience and have not received any training on brain-based learning. They have shared that they are aware of the psychological association with learning but they cannot practice it in the classroom and still they rely on direct teaching.

\section{Special Education for the students with Reading disabilities}

Participant 11, a special education teacher said, that there is an insufficiency for special education teachers in Tamil Nadu. They added that all schools are not equipped with special education teachers. Each special education teacher has to supervise nearly 10 to 15 schools as a result, they did not have sufficient time to meet students at regular intervals. Participant 12 mentioned that special education teachers were simply tutors to the students with reading disabilities who help them in revising the lessons and they seldom provide sensory and cognitive strategies for such students to overcome reading disability. Participant 13 reported that collaboration with the mainstream teachers for teaching the students with reading disability is not possible, because their main concern is students with other disabilities like visual impairment, hearing impairment and Autism. Participant 14 informed that they hardly receive training to teach students with reading disability and similarly they seldom provide training to mainstream teachers on general inclusive education.

The perceptions and the responses from the teachers were further segregated as subthemes in the following table to derive an appropriate result for the study. 

Inclusive learning strategies to enhance reading skill among the students with reading disability: An Occupation and Participation Approach to Reading Intervention (OPARI) in the rural Indian classroom

\begin{tabular}{|c|c|c|c|}
\hline \multirow[t]{2}{*}{ Subthemes } & \multirow{2}{*}{$\begin{array}{l}\text { No. of } \\
\text { Participant }\end{array}$} & \multicolumn{2}{|c|}{ No. of Responses } \\
\hline & & Yes & No \\
\hline 1. The class strength is huge & 14 & 5 & 9 \\
\hline 2. Sufficient knowledge of reading disability & 14 & 4 & 10 \\
\hline 3. Trained to teach reading disabilities & 14 & 1 & 13 \\
\hline 4. Providing appropriate strategies for the RD & 14 & 3 & 11 \\
\hline 5. Aware of neurocognitive teaching strategies & 14 & 0 & 14 \\
\hline 6. Supervising the RD at regular intervals & 14 & 3 & 11 \\
\hline $\begin{array}{l}\text { 7. Reading disabilities are either poor or average } \\
\text { learners }\end{array}$ & 14 & 9 & 5 \\
\hline 8. Special Education is needed to them & 14 & 6 & 8 \\
\hline 9. The primary concern is reading disability & 14 & 0 & 14 \\
\hline $\begin{array}{l}\text { 10. Collaboration with special education teachers is } \\
\text { successful }\end{array}$ & 14 & 0 & 14 \\
\hline 11. Reading Aids for reading disabilities are given & 14 & 0 & 14 \\
\hline
\end{tabular}

(Table1: Perceptions and responses of the teachers towards reading disability)

The above table is the evidence that teachers have low awareness of dyslexia and they are less equipped for teaching reading disability. The teachers have not been given any training to teach the students with reading disability and the efforts taken by the teachers to teach students with reading disability is not enough to overcome the disability. Each school does not have special education teachers therefore the collaboration of special education teachers with mainstream teachers is demanding. Reading disability has not been considered as a serious learning disability and early intervention has not been given to the students with reading disability whereas students with other disabilities receive early intervention to some extent.

\section{Discussion}

The results are the indication that the teaching methods for students with reading disability do not receive pedagogical and clinical intervention and so rural schools in Tamil Nadu require therapeutic associative teaching for students with reading disability. To answer, "what could be an effective intervention to overcome reading disabilities?", the study recommends school-based occupational therapy. The reason to implement occupational therapy is that it is the only form that provides intervention in a pleasurable way, strengthens cognition and neurorehabilitation. As mentioned earlier, occupational therapy is based on the principle of pleasure that helps to adapt 
the expected behaviour or condition through regular activities. With regard to reading intervention, occupational therapy recommends the following tools as a preferred medium for reading: laptop, television, tabs, handouts, newspaper and magazine. Through these reading tools, the teacher could identify the preferred tool of the learner. Specific to reading disability, occupational therapy introduces an exclusive approach for reading called Occupation and Participation Approach to Reading Intervention (Grajo \& Candler, 2016a). This approach is a thorough intervention for reading disabilities because the strategies that the approach enfolds have the credibility to establish better cognition and neurorehabilitation. Occupation and Participation Approach to Reading Intervention (OPARI) is an approach to adapt reading skill and its five principles are: engaging students in reading, providing self-learning strategies to the children, using various context for reading, collaboration with teachers and parents and facilitating a sense of mastery and competence that would increase participation in reading (Grajo \& Candler, 2016b). The study highlights three major issues regarding reading disability, for which the solution has been approached through OPARI and is discussed as follows;

Issue 1: The participants said that, for the reading disabilities, they choose certain units in the text and use rote learning as a strategy to learn the lessons, which is not a recommended strategy for comprehensive learning. The study has identified that the use of memorizing the lessons has restricted the sensory and other cognitive strategies for learning and as a result, the children with reading disability cannot overcome the struggles in reading and continue to be reading disabled. OPARI as an approach to reading intervention insists on employing different strategies that would nurture reading. Specifically, OPARI recommends sensory skills and cognitive skills, because they are the driving source for reading. Visual and cognitive cues are much needed for reading comprehension. In OPARI, through the preferred reading tool, the readers could activate their sensory and cognitive skills; the foremost of sensory skills for reading are visual sensory skills that function in visual alertness, visual memory, and visual discrimination and are nurtured through the preferred reading material (Schneck, 2013). These visual strategies would help the students with reading disability to identify the letter and letter string, shapes, words; since the preferred reading medium is not a regular textbook or print material, the learners would feel comfortable with the letters, text format, style, colour, the background of the letters while reading. On the other hand, the preferred reading medium enhances cognitive skills like phonological processing, attention, memory, and retrieval that are required for reading to take place. The teacher could provide the preferred reading medium according to the readers' interest; providing the preferred reading medium at the early intervention stage would strengthen the sensory activities at the early reading period and nourish cognitive skills at the secondary reading period (Copley et al., 2008). The participants have also informed that the classrooms are equipped with ICT tools like projectors, audio recorders, computers and tabs but these ICT tools are not employed for reading, instead, they are used for conceptual teaching in science. These ICT tools are teacher-centred where the students are passive users. Through OPARI, students can be converted into active users of ICT as OPARI breaks the stereotypical method of reading i.e., reading through textbooks or print mediums, and widens the choice of reading by providing the preferred reading medium or tools for reading. The teacher can engage the students with reading disability in the reading participation by converting the regular curriculum to the ICT tools; Learning through digital medium like tab, television, phones, smart classroom will motivate them which will further 
generate self-learning strategies in the reader. Apart from the regular curriculum, reading could be cultivated through the use of various contexts. The conviction of OPARI is that various contexts would nourish the reading skill; Contexts like a classroom, community library, friends or peer environment would provide greater spaces for reading (Grajo \& Candler, 2016a). Teacher should create such context for reading because encountering various context would enrich comprehension skills and better understanding, it would enable the students to use newly acquired words in appropriate situations

Issue 2: Few participants stated that they practice peer teaching in their classroom because, in the rural academic environment, students share most of their academic performance with the teachers, their peers and the participation of parents being minimal. Therefore, they encourage peer teaching but it is not practised for reading improvement instead peer teaching in the Indian context involves in conceptual teaching of science studies and mathematics. To overcome reading difficulties, OPARI suggests peer teaching or peer influence in the rural classrooms. OPARI believes that observing the reading skill and socializing with peers would reduce the fear of reading among students with reading disability. The better reading performance takes place in an emotion-free zone; The anxiety level of the readers will be reduced while providing or creating a pleasurable learning environment (Ujiie \& Krashen, 2002). Therefore, learning along with the peers motivate the students with reading disabilities to learn more, reduce fear and anxiety towards reading. Reduced affective emotions and increased optimistic emotions would improve self-motivation and direct the students towards reading achievement (Tyng et al., 2017). If the rural Indian classrooms engage peer teaching meaningfully, better reading performance would be achieved by students with reading disabilities.

Issue 3: The participants shared that they were unaware of the neurocognitive strategies or the neuroscience behind the education. OPARI provides appropriate sensory, cognitive strategies, peer teaching and various contexts that help in adapting the reading, which further instigate neuroplasticity (Ayres, 1972; Schaaf \& Miller, 2005; K. J. Schkade \& Schultz, 2003) and thus indirectly OPARI becomes the source of neurorehabilitation and it is perfect to make the claim that OPARI is a holistic intervention to reading. The participants should recall the fact that OPARI is an intervention based on occupational therapy where occupation is provided through the preferred reading medium. With regard to occupational therapy, significant changes have been observed in the brain's rewarding system. For example, Occupational therapy has rehabilitated stroke patients and significant changes have been observed in the neural mechanism. Similarly, occupational adaptation using drawing and music has induced dopamine and facilitated the patients with aphasia (Gutman \& Schindler, 2007).

Based on these evidences, the article designs the theoretical model of neurocognition while adapting reading through the OPARI approach. The article takes an effort to justify that OPARI stimulates and enhances neurocognition through the theoretical neural plasticity model. Since the participants were unaware of neurocognition through teaching pedagogies, the article attempts to prove to academicians that OPARI is an explicit intervention and learning pedagogy for reading disability. This article tries to answer the question, "How teaching instructions would affect neurocognition?". As mentioned earlier, the principle behind OPARI is driven by a factor of pleasure that aims to achieve the expected behaviour by employing regular, daily activities. When 
reading is established through the preferred reading medium, the readers would attain a state of pleasure. The state of pleasure drives readers to read extensively which brings changes in the neural network. A question may arise on the functions or changes in the brain (i.e) "what happens in the brain when reading is adapted through OPARI?". To answer this, and to explain the abstract of neuroplasticity, the article puts forth dopamine, a neurochemical in the reward centre of the brain, and it is noted as one of the key neurotransmitters to respond to the pleasure and reward activities. Dopamine plays a major role in responding to novel stimulus, rewards and helps in adopting a behaviour. Pleasurable activities determine the dopamine level in the mesocorticolimbic pathway that connects the ventral tegmental area in the midbrain to the ventral striatum of the basal ganglia in the forebrain. The dopaminergic pathway is closely associated with the prefrontal cortex of the human brain. The prefrontal cortex, nucleus accumbens, the amygdala is collectively called the mesocorticolimbic pathway that supports the cognitive and emotional stimulus (Gutman \& Schindler, 2007; Wong et al., 2012).

OPARI cultivates reading, a form of procedural learning that has rules for grapheme and phoneme identification, association and discrimination, rules of grammar, word and sentence combination and during the procedural learning the brain regions namely, the basal ganglia, prefrontal cortex (Kropotov \& Etlinger, 1999) and inferior frontal gyrus (Ullman, 2004; Wong et al., 2012) are activated. Participating in reading skills would activate the above-mentioned areas which specifically comes under the dopaminergic system. Secondly, the Amygdala, one of the regions in the mesocorticolimbic pathway is found to be associated with emotion regulation. Regulation of emotion is crucial in any learning and behaviour modification and on the other side peer teaching in OPARI would control negative emotions towards learning and motivate the students with reading disability towards competence in reading. Affecting factors or affective emotions in learning such as fear, low self-esteem, anxiety would be alleviated by offering selflearning strategies to the students allowing students to pursue with a comfortable emotion in learning. The self- learning strategies of OPARI regulates the amygdala region of the brain to synthesize optimistic emotions in learning. Finally, OPARI allows the readers to adapt reading skills through a preferred reading medium and the adapted reading skill induces the dopamine in the brain's rewards system or the mesocorticolimbic pathway that results in pleasure reading. The pleasure reading provokes the reader to the next level of reading skill where the reader becomes an extensive reader; the extensive reading calls for neural changes and it helps in better cognition. As a result of extensive and desired reading, the reader enriches her/his vocabulary, comprehension, logical thinking, and writing style (Ponniah \& Priya, 2014). This process of attaining reading through occupation generates a cyclic process.

\section{Pleasure reading $\longleftrightarrow$ Dopamine activation}

Reading increases dopamine and in turn, dopamine stabilizes the language areas where further reading takes place. Thus, the abstract of neural plasticity puts forth the notion that dopamine in the mesocorticolimbic region enriches reading on practising OPARI. At the peripheral level, this article justifies the theory of neurocognition through OPARI and also creates an awareness among the participants on the association between education and neuroscience.

The illustration below explains the neural plasticity through Occupation and Participation Approach to Reading Intervention. 
9 Inclusive learning strategies to enhance reading skill among the students with reading disability: An Occupation and Participation Approach to Reading Intervention (OPARI) in the rural Indian classroom

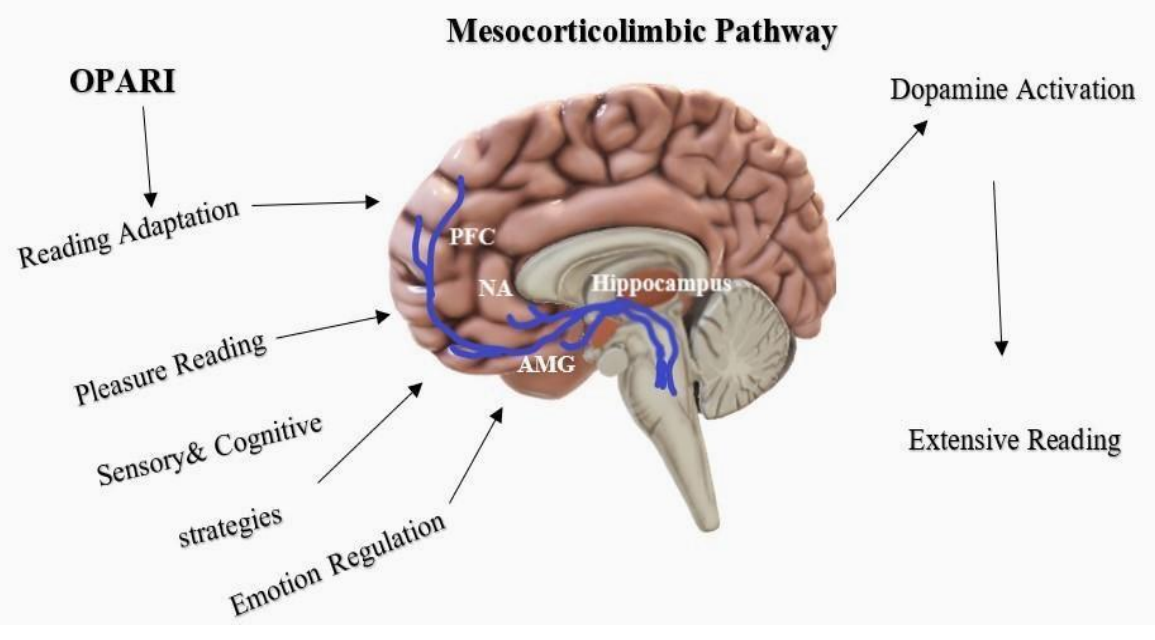

(Fig.1: OPARI working model in the mesocorticolimbic region)

Reading is an unremitting activity carried throughout life, through which an individual's visual skill, vocabulary, creativity and comprehending skills are strengthened; Reading briefly modifies the internal and external behaviour (Dehaene et al., 2015). OPARI is designed for students with a reading disability, who lack the above-mentioned skills but is not a complete clinical intervention to eradicate reading difficulties. This approach stimulates reading for students with reading disabilities through the external and internal factors of the reader. OPARI aims to improve reading in a pleasurable way therefore reading cannot be forcefully implemented among the reading disabilities. OPARI is also known as a reader-centred approach and therefore to cultivate reading, the teachers have to willingly execute OPARI for the benefit of reading disabilities. Based on the above- mentioned discussions, if OPARI is executed, students with reading disabilities would not feel or exhibit their reading difficulty as they would have their preferred reading tool to develop their reading skill.

\section{Conclusion}

To stimulate reading ability in students with a reading disability, OPARI should be implemented in the Indian classroom. Though complete recovery is not possible for students with reading disabilities, occupational therapy would stabilize their capacity for reading. In the absence of clinical intervention for reading disability, early intervention employing occupational therapy at the schools would ameliorate reading skills. The following are some of the suggestions for the betterment of reading disabilities in the rural Indian Classrooms.

- To employ occupational therapeutic teaching to all children at least at the primary education level.

- To practice OPARI, rural Indian classrooms should be designed with twenty-five students per class and the appropriate tools for reading should be provided to the students. 
- Teachers should receive training on brain-based teaching and learning strategies.

- Each school must have a special education trainer

Considering these suggestions, if occupational therapeutic teaching and learning are to be executed then the integrated education scheme would be highly successful in the rural Indian Classroom.

\section{Reference}

Ayres, A. (1972). Sensory Integeration and Learning disorders. Western Psychological Services.

Baker, L., \& Wigfield, A. (1999). Dimensions of Children's Motivation for Reading and Their Relations to Reading Activity and Reading Achievement. Reading Research Quarterly, 34(4), 452-477. https://doi.org/10.1598/rrq.34.4.4

Bissell, J., \& Cermak, S. (2015). Frameworks, Models and Trends in School-Based Occupational Therapy in the United States. The Israeli Journal of Occupation Therapy, 24(2-3), 49-69. https://www.researchgate.net/publication/279538801_Frameworks_Models_and_Trends_in_SchoolBased_Occupational_Therapy_in_the_United_States\%5Cnhttp://www.isot.org.il/Uploads/Attachment s/37256/school_based_occupational_therapy.pdf

Copley, J., Nelson, A., Turpin, M., Underwood, K., \& Flanigan, K. (2008). Factors influencing therapists' interventions for children with learning difficulties. Canadian Journal of Occupational Therapy, 75(2), 105-113.

Dehaene, S., Cohen, L., Morais, J., \& Kolinsky, R. (2015). Illiterate to literate: Behavioural and cerebral changes induced by reading acquisition. Nature Reviews Neuroscience, 16(4), 234-244. https://doi.org/10.1038/nrn3924

Frolek Clark, G. (2016). The occupations of literacy: Occupational therapy's role. Journal of Occupational Therapy, Schools, and Early Intervention, 9(1), 27-37. https://doi.org/10.1080/19411243.2016.1152835

Grajo, L. C., \& Candler, C. (2016a). An Occupation and Participation Approach to Reading Intervention (OPARI) part I: Defining reading as an occupation. Journal of Occupational Therapy, Schools, and Early Intervention, 9(1), 74-85. https://doi.org/10.1080/19411243.2016.1141082

Grajo, L. C., \& Candler, C. (2016b). An Occupation and Participation Approach to Reading Intervention (OPARI) part II: Pilot clinical application. Journal of Occupational Therapy, Schools, and Early Intervention, 9(1), 86-98. https://doi.org/10.1080/19411243.2016.1141083

Gutman, S. A., \& Schindler, V. P. (2007). The neurological basis of occupation. Occupational Therapy International, 14(2), 71-85. https://doi.org/10.1002/oti.225

Kropotov, J. D., \& Etlinger, S. C. (1999). Selection of actions in the basal ganglia-thalamocortical circuits: Review and model. International Journal of Psychophysiology, 31(3), 197-217. https://doi.org/10.1016/S0167-8760(98)00051-8

Mu, K., \& Royeen, C. (2004). Facilitating participation of students with severe disabilities: Aligning schoolbased occupational therapy practice with best practices in severe disabilities. Physical and Occupational Therapy in Pediatrics, 24(3), 5-21. https://doi.org/10.1300/J006v24n03_02 
Occupational Therapy Practice Framework: Domain \& Process (Third Edit). (2014). American Occupational Therapy Association.

Ponniah, R. J., \& Priya, J. (2014). Pleasure reading and the acquisition of second language by adult ESL students. The International Journal of Foreign Language Teaching, 9(1), 16-22.

Sanocki, T., \& Dyson, M. C. (2012). Letter processing and font information during reading: Beyond distinctiveness, where vision meets design. 132-145. https://doi.org/10.3758/s13414-011-0220-9

Schaaf, R. C., \& Miller, L. J. (2005). Occupational therapy using a sensory integrative approach for children with developmental disabilities. Mental Retardation and Developmental Disabilities Research Reviews, 11(2), 143-148. https://doi.org/10.1002/mrdd.20067

Schkade, J. K., \& Schultz, S. (1992). Occupational Adaptation: Toward a Holistic Approach for Contemporary Practice, Part 1. American Journal of Occupational Therapy, 829-837.

Schkade, K. J., \& Schultz, S. (2003). Occupational adaptation: Perspectives in human occupation: Participation in life (P. Kramer, J. Hinojosa, \& C. . Roydeen (Eds.)). MD: Lippincott Williams \& Wilkins.

Schneck, C. (2013). Best practices in visual perception and academic skills to enhance participation. In F. Clark G \& B. Chandler E (Eds.), Best practices for occupational therapy in schools (pp. 431-442). American Occupational Therapy Association.

Shetty, A., \& Rai, B. S. (2014). Awareness and Knowledge of Dyslexia among Elementary School Teachers in India. 2(5), 1135-1143.

Singh Charan, G., \& Kaur, H. (2017). A Cross-Sectional Survey to Assess the Knowledge and Attitude Regarding Dyslexia among Teachers at Selected Schools, Punjab. International Journal of Science and Healthcare Research (Www.Gkpublication.In), 2(September), 9. www.gkpublication.in/ijshr.html

Ujiie, J., \& Krashen, S. D. (2002). Home Run Books and Reading Enjoyment. Knowledge Quest, 31 (1).

Ullman, M. (2004). Contributions of memory circuits to language: The declarative/procedural model. Journal of Cognition, 92, 231-270.

Wong, P. C. M., Morgan-Short, K., Ettlinger, M., \& Zheng, J. (2012). Linking neurogenetics and individual differences in language learning: The dopamine hypothesis. Cortex, 48(9), 1091-1102. https://doi.org/10.1016/j.cortex.2012.03.017

Zari, J., \& Hasselhorn, M. (2021). Orthographic knowledge predicts reading and spelling skills over and above general intelligence and phonological awareness. 21-4 